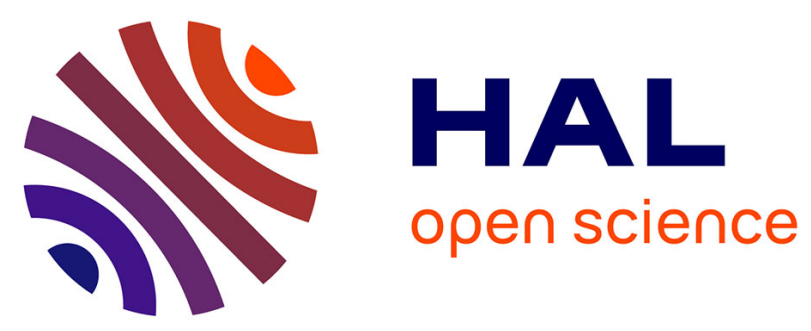

\title{
A flow split test to discriminating between heterogeneous and homogeneous contributions in Suzuki coupling
}

\author{
Amine Bourouina, Valérie Meille, Claude de Bellefon
}

\section{- To cite this version:}

Amine Bourouina, Valérie Meille, Claude de Bellefon. A flow split test to discriminating between heterogeneous and homogeneous contributions in Suzuki coupling. Journal of flow chemistry, 2018, 8 (3-4), pp.117-121. 10.1007/s41981-018-0020-7 . hal-02094809

\section{HAL Id: hal-02094809 \\ https://hal.science/hal-02094809}

Submitted on 26 Mar 2020

HAL is a multi-disciplinary open access archive for the deposit and dissemination of scientific research documents, whether they are published or not. The documents may come from teaching and research institutions in France or abroad, or from public or private research centers.
L'archive ouverte pluridisciplinaire HAL, est destinée au dépôt et à la diffusion de documents scientifiques de niveau recherche, publiés ou non, émanant des établissements d'enseignement et de recherche français ou étrangers, des laboratoires publics ou privés. 


\title{
A flow split test to discriminating between heterogeneous and homogeneous contributions in Suzuki coupling
}

\author{
Amine Bourouina · Valérie Meille* . Claude de \\ Bellefon*
}

Received: date / Accepted: date

\begin{abstract}
The homogeneous vs heterogeneous contributions when using solid catalysts for the Suzuki-Miyaura coupling is still disputed. Leaching is often observed and quantified albeit with unclear conclusions about contributions of the leached species and of the solid catalyst to the global catalytic activity. In this work, a new flow reactor design to discriminate both contributions is proposed. With the help of a simple reactor model, it has been possible to conclude that the coupling of 4iodoacetophenone with phenylboronic acid proceeded with the leached homogeneous species only, whatever the solid Pd/silica used, whereas chloro-derivatives behaves differently. This reactor is simple to build and could be of general use to reveal actual heterogeneous vs homogeneous catalysis for many reactions.
\end{abstract}

Keywords Suzuki-Miyaura $\cdot$ Heterogeneous $\cdot$ Leaching $\cdot$ Palladium

The knowledge of the reaction location is of prime importance both to design efficient industrial processes and to understand the underlying fundamental mechanisms. For example, many catalytic processes are operated with heterogeneous (solid) catalysts for easier separation and downstream treatments thus to lower operating costs. However, when the fluid phase is a liquid, it appears that the knowledge of the reaction location is not straightforward. Indeed, active catalytic species may leach from the solid, which is then a simple catalyst precursor, and solubilizes in the liquid phase in which it catalyzes the reaction. Several papers including recent reviews have analyzed such situations for many different type of catalytic processes.[1,2] One must realize that the design of a catalytic reactor is largely based on the assumption of the reaction location simply because the reaction time, i.e. the contact time between the

Université de Lyon, Institut de Chimie de Lyon, Laboratoire de Génie des Procédés Catalytiques (UMR 5285 CNRS-Université Lyon 1-CPE Lyon), 43 Bd du 11 Novembre 1918, 69616 Villeurbanne, France. . Valérie Meille

Tel.: +33-4-72431755

E-mail: vme@1gpc.cpe.fr

Claude de Bellefon

Tel.: +33-4-72431754

E-mail: cdb@lgpc.cpe.fr 
reagents and the catalyst, hence the reactor efficiency depends on that knowledge. Other characteristic of the reactor are also based on that assumption such as mass and heat transfer performances and mixing. Last, downstream treatments also depend on this knowledge, a simple distillation could transformed into a catalytic reactor producing side products when catalytic species are transported. Among the very large diversity of catalytic reactions, some are well known to be heterogeneous, i.e. processes in which the reaction takes place at the solid, but other are more questionable. $\mathrm{C}-\mathrm{C}$ coupling such as the Heck and Suzuki- Miyaura reactions are striking examples of this situation and have been the matter of extensive investigations since 20 years to actually demonstrated the heterogeneous (solid) vs homogeneous (liquid phase) catalysis.[3,4] With the wider use of continuous processes (Flow Chemistry), this issue has gained more interest since soluble catalytic species will be transported out of the reactor (C-C coupling, [5, 6] olefin metathesis, [7, 8] hydroformylation [9]). Indeed, whereas it is clear that the Heck reaction proceeds through a homogeneous mechanism in the liquid phase (even when starting from a solid catalyst precursor), the situation is much more complex for the Suzuki-Miyaura reaction. A review depicts the different approaches and techniques used to distinguish between homogeneous and heterogeneous Suzuki catalysis.[10] Protocols such as the correlation of Pd content in solution with the reaction yield,[11] the hot filtration tests,[2] the Three-Phase test,[12] poisoning tests [13,2] or the Nanoparticle-Exclusion Experiments [14] can be used. Heavier methods have been used also such as X-ray absorption spectroscopy (XAS) to monitor the structure of Pd NPs during the Suzuki reaction,[15] and Pd NP coated AFM tip.[16] Detailed discussions of the advantages and drawbacks of these methods have been published and the general conclusion emphasized that there is no single definitive experiment for making this distinction.[1, 17]

While everybody will agree with this general statement and would use a combination of methods before arriving at a compelling conclusion, it prompted us to reinvestigate ligand-free Pd SM coupling catalytic species with a new reactor design able to discriminate between homogeneous and heterogeneous catalysis. This new design is based on a continuous operation and avoids all the difficulties linked to the hot-filtration and the ambiguous exploitation of poisoning tests. At this point, we would like to mention that this method has been recently proposed by Barreiro et al. with the name "Tandem flow reactor". It was used for the Heck coupling [18] which is already known to proceed by homogeneous catalysis. As mentioned, this new method is based on the time-length analogy between closed (batch) reactors and open (continuous) reactors $[19,20]$ and simply adapts the well-practiced "Batch Split Test" (generally called "hot-filtration test" to a "Flow Split Test" (Figure 1). Doing so, this new method avoids the main drawback of the split test by ensuring a non-disruptive process between the heterogeneous liquid-solid reactor and the homogeneous "split" test.

In the batch split test, at a given reaction time $\mathrm{t}$, the reaction mixture is sampled (filtered) with caution to minimize or, better, avoid any solid catalyst in the sample. Then the sample is maintained under the same operating conditions than the original reaction mixture. The conversions (yields) are then measured in both reactors and compared. Three type of behavior could be observed: 
Batch Split Test

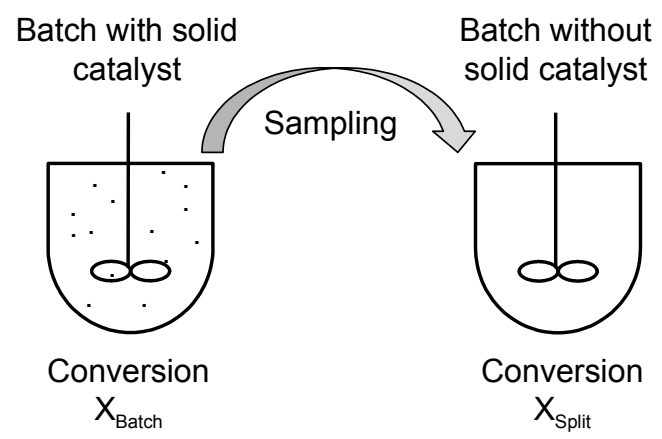

\section{Flow Split Test}

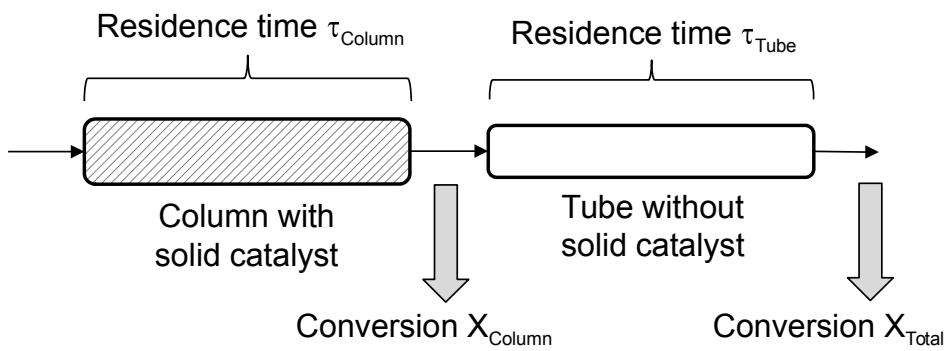

Fig. 1 Principle of split tests

- The conversion in the split reactor is unchanged compared to that at the time of sampling. In such case it is concluded that the reaction is occurring at the solid. The catalysis is heterogeneous.

- The conversion in the split reactor is increasing at comparable rate than the original batch reactor. In that case, it is concluded that the catalysis occurred in the liquid phase, albeit with no conclusion about the exact nature of the catalyst which could be nanoparticles or molecular species or both ...

- Any in-between situation for which it is difficult to assess the location of the catalytic reaction.

In the flow split test, the liquid mixture is pumped in a first reactor containing the putative solid catalyst for a given contact time then, it is circulated in an empty tube. While the three types of behavior could be observed very much like for the batch split test, this set-up presents many interesting features to help distinguishing between solid or liquid phase catalysis that cannot be achieved in the Batch mode. Some of these features are detailed below:

- The "split" between the solid containing reactor and the empty section is performed with no risk of exposition of the reaction mixture to air, moisture, etc. and without temperature variations that could impact the reaction.

- The amount of solid (putative) catalyst in the reactor is very high so that even in the situation where a small amount of solid would be entrained, the amount of conversion 
due to that solid in the empty tube section would be negligible.

- The flow split test is most often operated in the steady-state regime thus revealing unambiguously any unsteady-state processes such as catalyst deactivation that could lead to false conclusions in the batch mode.

- The reaction time in the solid containing section and that in the empty tube section can be varied independently, precisely and easily by changing the liquid flow rate and/or the length of the tube/column.

- Simple reactor modelling could provide the potential activity of the solid and that of liquid phase species.

Thus the flow split test is a promising concept that has a very broad scope for application and which, when coupled to simple reactor modelling has the potential to build in depth and unambiguous conclusions concerning the homogeneous vs heterogeneous nature of catalysis.

In order to demonstrate the efficiency of the flow split test concept, the SuzukiMiyaura (SM) reaction was chosen. For the SM reaction, the homogeneous (liquid phase) vs heterogeneous (solid) nature of the reaction mechanism is indeed still under debate in the literature. Also, Suzuki-Miyaura reactions are of increasing importance for organic synthesis and represent up to $10 \%$ of a 3 million patents database in the field of pharmaceuticals as pointed in a recent paper reporting the use of intelligent search algorithms.[21] From an industrial viewpoint, the idea to catalyze the SM reaction with insoluble Pd catalysts is very attractive either in the form of palladium nanoparticles containing solids (Pd/alumina, $\mathrm{Pd} / \mathrm{silica}, . .$.$) or anchored pal-$ ladium transition metal complexes on solids which then are easily separated from the reaction mixture by conventional processes such as filtration or decantation for example. Consequently, a huge effort has been made to prepare, characterize and test such catalysts with very probing results.[22,23,24,25,26,27] Meanwhile, it has also generated a controversial debate since in many instances, soluble Pd species where identified thus calling for less robust conclusion about "true" heterogeneous catalysis.[28,29] Last, many reports point Pd leaching from the solid thus calling for expensive downstream processing to comply with regulations.

The SM coupling of 4-iodoacetophenone with phenyl boronic acid in ethanol in the presence of Pd containing solids and sodium methanolate was selected as the target reaction. Three commercially available Pd containing solids were tested: Siliacat Pd(0) (Silicycle R815-100), Siliacat Pd(II)-DPP (Silicylcle R390-100), and Strem $\operatorname{Pd}(0)$ (Strem Chemicals 46-2090). The catalytic columns were prepared using stainless steel tubes $(4.6 \mathrm{~mm}$ i.d.) with different lengths. The solids were packed in the tubes manually in a similar fashion than HPLC columns. Both ends of the column were fitted with appropriate Swagelok fittings, including filters. The empty tube section was made from PFA tube $(1.7 \mathrm{~mm}$ i.d., $1 \mathrm{~m}$ length). Note here that each column could be used for several experiments, i.e. several flow rates.

A typical reactant solution was prepared by dissolution of 4-iodoacetophenone ( 1 eq, $0.25 \mathrm{~mol} / \mathrm{L}$ ), phenylboronic acid (1.2 eq.), $\mathrm{MeONa}(1.5$ eq.) and $\mathrm{n}$-dodecane (standard for GC, 0.3 eq.) in ethanol. The solution was pumped through the preheated $60{ }^{\circ} \mathrm{C}$ catalytic column and then through the empty tube (Figure 2). Samples were withdrawn and quenched in a mixture of water $(2 \mathrm{~mL})$ and dichloromethane $(1 \mathrm{~mL})$. The organic phase was analyzed using GC-FID chromatography (details in ESI). 


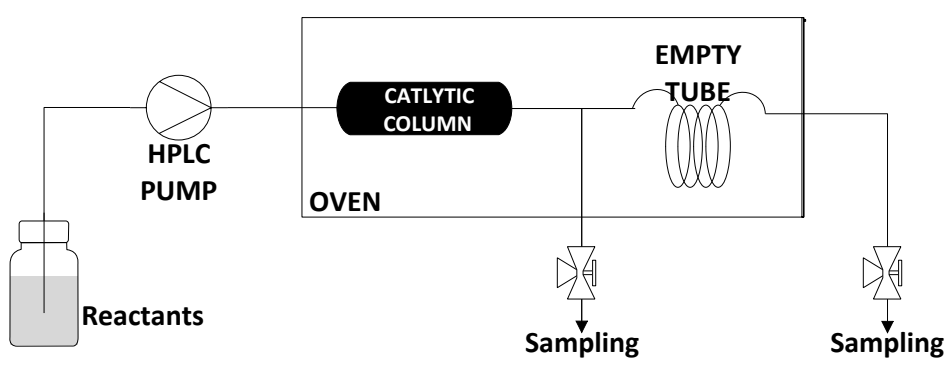

Fig. 2 Experimental device

Results obtained with two catalytic columns are presented in Figure 3.

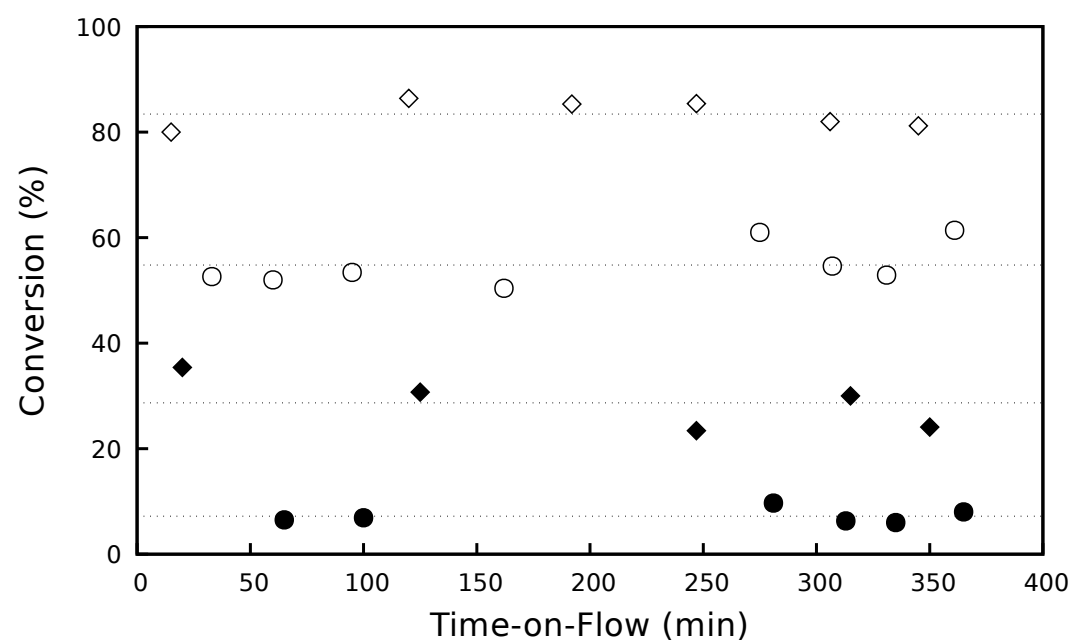

Fig. 3 Conversion of 4-iodoacetophenone at the column outlet (filled symbols $\bullet$ ) and at the tube outlet (empty symbols $\bigcirc \diamond$ ) for 2 catalysts. The dotted lines represent the mean value for each series. $1.8 \mathrm{mg} \mathrm{Pd}$ for Siliacat $\operatorname{Pd}(0) \bigcirc \bullet$, and $15 \mathrm{mg} \operatorname{Pd}$ for Strem $\operatorname{Pd}(0) \diamond \diamond$; Temperature of $60^{\circ} \mathrm{C}$ and liquid flow rate of $0.3 \mathrm{~mL} / \mathrm{min}$ for both catalysts in both sections.

Note that the absence of any possible deposition of active $\mathrm{Pd}$ inside the empty tube was verified, by flowing reactants directly through the preheated tube. The empty symbols show the conversion measured at the end of the empty tube, representing the total conversion reached in both successive zones (filled column + empty tube). The conversion increase during residence in the empty tube is thus due to palladium that has leached from the column. The test was long enough $(6 \mathrm{~h})$ to inform about the stability of the conversion. If Pd leaching was due to badly anchored Pd species on the solid surface, a rapid decrease of the activity would have been expected. Other experiments with 4-iodoacetophenone are presented in Table 1 (entries 1-11) for the three catalysts. The columns present the conversion and yield after the column section and at the end of the tube section (sum of both sections), and the corresponding residence times. Note that the residence time in the tube section is much longer than that in the 
Table 1 All flow split tests- halogenoacetophenone concentration $0.25 \mathrm{~mol} / \mathrm{L}, 60^{\circ} \mathrm{C}$ for iodo, $67^{\circ} \mathrm{C}$ for chloro - *inerted with nitrogen

\begin{tabular}{|c|c|c|c|c|c|c|c|c|}
\hline Entry & Halogen & Catalyst & $\begin{array}{l}m_{P d} \\
(\mathrm{mg})\end{array}$ & $\begin{array}{l}\text { Conversion (yield) } \\
\text { After column }\end{array}$ & $\begin{array}{l}\text { Conversion (yield) } \\
\text { Total after tube }\end{array}$ & $\begin{array}{l}\tau_{\text {Column }} \\
(\mathrm{min})\end{array}$ & $\begin{array}{l}\tau_{\mathrm{Col}+\text { tube }} \\
(\mathrm{min})\end{array}$ & $\begin{array}{l}\text { Pd leach. } \\
\text { ppm }\end{array}$ \\
\hline 1 & \multirow{11}{*}{ I } & \multirow{5}{*}{ Siliacat $\operatorname{Pd}(0)$} & \multirow{5}{*}{8.7} & $50(50)$ & $100(100)$ & 2.1 & 12.8 & \multirow{5}{*}{0.12} \\
\hline 2 & & & & $21(21)$ & $58(58)$ & 1.05 & 6.45 & \\
\hline 3 & & & & $10(10)$ & $43(43)$ & 0.7 & 4.3 & \\
\hline 4 & & & & $5(5)$ & $23(23)$ & 0.42 & 2.56 & \\
\hline 5 & & & & $3(3)$ & $13(13)$ & 0.3 & 1.8 & \\
\hline 6 & & \multirow{4}{*}{ Strem $\operatorname{Pd}(0)$} & \multirow{4}{*}{15} & $58(58)$ & $100(100)$ & 2.1 & 12.8 & \multirow{4}{*}{0.1} \\
\hline 7 & & & & $24(24)$ & $56(56)$ & 0.7 & 4.3 & \\
\hline 8 & & & & $12(12)$ & $46(46)$ & 0.42 & 2.56 & \\
\hline 9 & & & & $15(15)$ & $38(38)$ & 0.3 & 1.8 & \\
\hline 10 & & \multirow{2}{*}{ Siliacat Pd(II)-DPP } & \multirow{2}{*}{0.6} & $56(54)$ & $95(88)$ & 0.05 & 5.45 & \multirow{2}{*}{0.53} \\
\hline 11 & & & & $37(32)$ & $75(70)$ & 0.02 & 2.16 & \\
\hline $12^{*}$ & \multirow{4}{*}{$\mathrm{Cl}$} & \multirow{2}{*}{ Siliacat $\operatorname{Pd}(0)$} & \multirow{2}{*}{17.5} & $100(41)$ & $100(41)$ & 0.83 & 2.14 & \multirow{2}{*}{ not available } \\
\hline $13^{*}$ & & & & $87(36)$ & $87(37)$ & 0.55 & 1.4 & \\
\hline 14 & & \multirow{2}{*}{ Siliacat Pd(II)-DPP } & \multirow{2}{*}{11} & $47(33)$ & $48(34)$ & 0.42 & 2.14 & \multirow{2}{*}{0.17} \\
\hline 15 & & & & $35(21)$ & $35(22)$ & 0.32 & 1.6 & \\
\hline
\end{tabular}

column to compensate for palladium concentration. The rows present different tests performed at various flow rates with a variety of catalysts and with 2 different halides. All results show a conversion increase in the tube section, which can be explained by palladium leaching. ICP/MS analysis of the reaction products (last column of Table 1), performed in a mixture of collected products at different flow rates, indeed showed a mean Pd concentration of ca. $100 \mathrm{ppb}$ for both $\mathrm{Pd}(0)$ catalysts and ca. $500 \mathrm{ppb}$ in the case of the immobilized complex (Siliacat Pd(II) DPP).

In order to identify the homogeneous or heterogeneous contributions of the catalysts (namely in the column where both can co-exist), a reactor model was build. The following assumptions were used: both reactors are isotherm - axial dispersion is neglected - first order rate laws with respect to reagents and catalysts with no effect of the base concentration - first order rate law for Pd leaching regarding the solid Pd and arylhalide reagent concentrations - no mass transfer limitations - negligible catalyst deactivation - possible adsorption of leached Pd species neglected. The justification of these assumptions, out of the scope of this paper, comes from a thorough analysis of the literature and/or by experiments performed at the team (rate laws, mass transfer,...).

The resulting model is presented in the supplementary material, leading to the following rate expressions of homogeneous (1), heterogeneous (2) and leaching (3) reaction rates:

$$
\begin{gathered}
r_{\text {hom }}=k_{\text {hom }} C_{A} \cdot C_{B} \cdot C_{P d}\left[\mathrm{~mol} / \mathrm{m}^{3} / \mathrm{s}\right] \\
r_{\text {het }}=k_{\text {het }} C_{A} \cdot C_{B} \cdot C_{P d-\text { solid }}\left[\mathrm{mol} / \mathrm{m}^{3} / \mathrm{s}\right] \\
r_{\text {leach }}=k_{\text {leach }} C_{A} \cdot C_{P d-\text { solid }}\left[\mathrm{mol} / \mathrm{m}^{3} / \mathrm{s}\right]
\end{gathered}
$$

The kinetic parameters obtained for 4-iodoacetophenone are presented in Table 2. Identical values were obtained for both $\operatorname{Pd}(0)$ catalysts. The heterogeneous contribution appears to be negligible for all the solid catalysts tested, even for Siliacat $\operatorname{Pd}(0)$ 
Table 2 Kinetic parameters for 4-iodoacetophenone

\begin{tabular}{llll} 
& $\begin{array}{l}k_{\text {hom }} \\
\left(\left(\mathrm{m}^{3}\right)^{2} / \mathrm{mol}^{2} / \mathrm{s}\right)\end{array}$ & $\begin{array}{l}k_{\text {het }} \\
\left(\left(\mathrm{m}^{3}\right)^{2} / \mathrm{mol}^{2} / \mathrm{s}\right)\end{array}$ & $\begin{array}{l}k_{\text {leach }} \\
\left(\mathrm{m}^{3} / \mathrm{mol} / \mathrm{s}\right)\end{array}$ \\
\hline $\begin{array}{l}\text { Siliacat Pd(0) } \\
\begin{array}{l}\text { Strem Pd(0) } \\
\text { Siliacat Pd(II)-DPP }\end{array}\end{array}$ & $0.31 \pm 0.02$ & $(2.5 \pm 1) \cdot 10^{-7}$ & $(3.84 \pm 0.04) \cdot 10^{-9}$ \\
& $0.31 \pm 0.05$ & $(4.15 \pm 0.38) \cdot 10^{-5}$ & $(3.81 \pm 0.09) \cdot 10^{-7}$
\end{tabular}

which is reported not to leach.[30,31] The validity of the model and the parameters obtained is demonstrated by the parity plot showing the good agreement between the computed and the experimental iodoacetophenone concentration for the 3 different catalysts at different flow rates (see ESI). Thus, it is clear that Pd concentrations as low as $100 \mathrm{ppb}$ are sufficient to run the reaction. This corresponds to a turnover frequency in the range $10^{5}-10^{6} \mathrm{~mol}_{\text {iodo }} / \mathrm{mol}_{P d} / \mathrm{h}$. Low concentration active Pd species were reported as early as in 2005. The group of Leadbeater [32] reported a smart experiment to proof that trace amount of $\mathrm{Pd}(50 \mathrm{ppb})$ found in commercially available sodium carbonate are responsible for the generation of the biaryl rather than, as previously suggested by the same group, an alternative non-palladium-mediated pathway. Thus, the SM coupling of aryl bromide with aryl boronic acids was reported to proceed efficiently at very low Pd loading (100 ppb) within short time (5 min) albeit at rather high temperature $\left(150^{\circ} \mathrm{C}\right)$. Later, other evidence for trace Pd catalysis of the SM coupling was also provided by the group of Lipshutz [33] with so called "Fe-ppm Pd nanoparticle" containing $320 \mathrm{ppb}$ Pd. These reports point the hyperactive nature of very low level Pd species and show that their activity relies on the way they are generated in the medium. Turnover numbers reached with the catalyst are provided in ESI (Table S2); after several hours, the TON with respect to all Pd atoms reaches only 4 , showing that the solid can act as a huge reservoir, allowing to provide a continuous production despite leaching.

Other experiments have been performed with 4-chloro- instead of 4-iodoacetophenone. The behaviour of the chloro compound is completely different from the iodo one: the amount of Pd leached is in the same order of magnitude but there is no extraactivity in the tube section (Table 1, entries 12-15). Moreover, the reaction is not selective and dehalogenation occurs, as also observed by others.[23] This selectivity aspect will have to be further investigated since some research groups show high selectivities.[34]

A novel set-up, the flow split test, allowing to quantify the homogeneous vs. heterogeneous contributions of Pd for the Suzuki coupling of 4-iodoacetophenone with phenylboronic acid is reported. The heterogeneous contribution is shown to be negligible for iodo derivatives. Future work will be dedicated to 4-chloroacetophenone, to get more data and try to explain its particular behavior with respect to the reaction mechanism. 4-bromoacetophenone will also be studied.

The flow split test is a simple and rather inexpensive set-up that could be an ideal work horse for helping chemists to differentiating between homogeneous and heterogeneous contribution in Suzuki-Miyaura coupling and many other reactions. 
Acknowledgements ED Chimie de Lyon is gratefully acknowledged for funding the $\mathrm{PhD}$ thesis of $\mathrm{A}$. Bourouina. CNRS, CPE Lyon and UCBL are also thanked for financial support.

\section{References}

1. J.A. Widegren, R.G. Finke, J. Mol. Catal. 191, 187 (2003)

2. N.T.S. Phan, M. Van Der Sluys, C.W. Jones, Adv. Synth. Catal. 348(6), 609 (2006)

3. A.J. Reay, I.J.S. Fairlamb, Chem. Commun. 51(91), 16289 (2015)

4. A.D. Zotto, D. Zuccaccia, Catal. Sci. Technol. 7(18), 3934 (2017)

5. D. Cantillo, C.O. Kappe, ChemCatChem 6(12), 3286 (2014)

6. C. Len, S. Bruniaux, F. Delbecq, V.S. Parmar, Catalysts 7(5), 146 (2017)

7. J. Lim, S. Seong Lee, J.Y. Ying, Chem. Commun. 46(5), 806 (2010)

8. J.L. Cheong, D. Wong, S. Lee, J. Lim, S.S. Lee, Chem. Commun. 51, 1042 (2015)

9. A.M. Kluwer, C. Simons, Q. Knijnenburg, J.I. van der Vlugt, B. de Bruin, J.N.H. Reek, Dalton Transactions 42(10), 3609 (2013)

10. A.F. Schmidt, A.A. Kurokhtina, Kinetics and Catalysis 53(6), 714 (2012)

11. S.S. Soomro, F.L. Ansari, K. Chatziapostolou, K. Köhler, J. Catal. 273(2), 138 (2010)

12. S.J. Broadwater, D.T. McQuade, J. Org. Chem. 71(5), 2131 (2006)

13. J.Y. Lee, R.J. Tzeng, M.C. Wang, H.M. Lee, Inorg. Chim. Acta 464, 74 (2017)

14. A.V. Gaikwad, A. Holuigue, M.B. Thathagar, J.E. ten Elshof, G. Rothenberg, Chem.-Eur. J. 13(24), 6908 (2007)

15. P.J. Ellis, I.J.S. Fairlamb, S.F.J. Hackett, K. Wilson, A.F. Lee, Angew. Chem. Int. Ed. 49(10), 1820 (2010)

16. J.J. Davis, Y. Hanyu, Nanotechnology 21(26), 265302 (2010)

17. M. Pérez-Lorenzo, J. Phys. Chem. Lett. 3(2), 167 (2012)

18. E.M. Barreiro, Z. Hao, L.A. Adrio, J.R. van Ommen, K. Hellgardt, K.K.M. Hii, Catal. Today 308, 64 (2018)

19. O. Levenspiel, Chemical Reaction Engineering - 3rd Ed. (Wiley \& Sons, 1999)

20. C. de Bellefon, "Catalytic engineering aspects of flow chemistry" in "Flow Chemistry: Fundamentals", 1st edn. (De Gruyter, Berlin ; Boston, 2014)

21. N. Schneider, D.M. Lowe, R.A. Sayle, M.A. Tarselli, G.A. Landrum, Journal of Medicinal Chemistry 59(9), 4385 (2016)

22. C. Baleizão, A. Corma, H. García, A. Leyva, Chem. Commun. 0(5), 606 (2003)

23. L. Artok, H. Bulut, Tetrahedron Lett. 45(20), 3881 (2004)

24. B. Yuan, Y. Pan, Y. Li, B. Yin, H. Jiang, Angew. Chem. Int. Ed. 49(24), 4054 (2010)

25. S.M. Islam, P. Mondal, A.S. Roy, S. Mondal, D. Hossain, Tetrahedron Lett. 51(15), 2067 (2010)

26. F. Siga, H. Temel, M. Aydemir, Y.S. Ocak, S. Pasa, A. Baysal, Appl. Catal. A 449, 172 (2012)

27. Y.M.A. Yamada, S.M. Sarkar, Y. Uozumi, J. Am. Chem. Soc. 134(6), 3190 (2012)

28. A. Corma, D. Das, H. García, A. Leyva, J. Catal. 229(2), 322 (2005)

29. T.P.N. Tran, A. Thakur, D.X. Trinh, A.T.N. Dao, T. Taniike, Appl. Catal. A 549(Supplement C), 60 (2018)

30. V. Pandarus, D. Desplantier-Giscard, G. Gingras, R. Ciriminna, P. Demma Carà, F. Béland, M. Pagliaro, Tetrahedron Lett. 54(35), 4712 (2013)

31. M. Pagliaro, V. Pandarus, F. Beland, R. Ciriminna, G. Palmisano, P.D. Cara, Catal. Sci. Technol. 1, 736 (2011)

32. R.K. Arvela, N.E. Leadbeater, M.S. Sangi, V.A. Williams, P. Granados, R.D. Singer, J. Org. Chem 70(1), 161 (2005)

33. S. Handa, J.D. Smith, M.S. Hageman, M. Gonzalez, B.H. Lipshutz, ACS Catalysis 6(12), 8179 (2016)

34. V. Pandarus, G. Gingras, F. Béland, R. Ciriminna, M. Pagliaro, Org. Process Res. Dev. 16(1), 117 (2012) 